\title{
On the distribution of zeros of solutions of a first order neutral differential equation
}

\author{
Baker FA, El-Morshedy HA* \\ Department of Mathematics, Faculty of Science, Damietta University, New Damietta 34517, Egypt
}

Received: 20 November 2014 / Accepted: 27 January 2015

* Corresponding author: (email: elmorshedy@yahoo.com)

\begin{abstract}
This paper is devoted to study the distribution of zeros of all solutions of the first-order neutral differential equation

$$
[x(t)-p x(t-T)]^{\prime}+Q(t) x(t-\sigma)=0, t>t_{0},
$$

where $p>1, \tau, \sigma>0$, and $Q \in C\left(\left[t_{0}, \infty\right),(0, \infty)\right)$.

We obtain new estimates for the distance between adjacent zeros of all solutions of the above equation under suitable criteria. Our results are supported with illustrative examples.
\end{abstract}

Keywords: Distribution of zeros; Oscillation, Neutral differential equations

\section{Introduction}

In this article, we estimate the distance between adjacent zeros of all solutions of the neutral equation

$$
[x(t)-p x(t-\tau)]^{\prime}+Q(t) x(t-\sigma)=0,
$$

where $\tau, \sigma>0, p>1$ and $Q \in C\left(\left[t_{0}, \infty\right),(0, \infty)\right)$. We shall assume that

$$
\int_{t-\tau}^{t} Q_{*}(s) d s \geq \zeta_{0}, \quad \text { for } t \geq t_{0}+2 \tau,
$$

for some $\zeta_{0}>0$ where $Q_{*}(t)=\min _{t-\tau \leq s \leq t}\{Q(s)\}$ for $t \geq t_{0}+\tau$. By a solution of Eq.(1) we mean a function $x \in C\left(\left[t_{0}-\lambda, \infty\right), R\right)$, where $\lambda=\max \{\sigma, \tau\}$, such that $x(t)-p x(t-\tau)$ is continuously differentiable and (1) is satisfied on $\left[t_{0}, \infty\right)$. We associate with (1) the initial condition $x(t)=\phi(t)$ on $\left[t_{0}-\lambda, t_{0}\right]$ where $\phi \in C\left(\left[t_{0}-\right.\right.$ $\left.\left.\lambda, t_{0}\right], R\right)$. The method of steps can be used to show that the resulting initial value problem has a unique solution. A solution $x$ is said to be oscillatory if it has arbitrary large zeros. Equation (1) is called oscillatory if all its solutions are oscillatory.
Neutral differential equations appear in many applications from engineering, physics, economy and mathematical biology see (Gopalsamy, 1992; Hale and Lunel, 1993; Kolmanovskii and Myshkis, 1999; Kolmanovskii and Nosov, 1986). The oscillation theory of neutral differential equations has received a great deal of attention in recent years; see (Agarwal et al., 2012; Bainov and Mishev, 1991; Erbe et al., 1995; Györi and Ladas, 1991) for an account of this theory. However, little is known about estimating the distance between consecutive zeros of the solutions of these equations; see (Wenrui et al., 2007; Wu et al., 2007; Wu and Xu, 2004; Yong and Zhicheng, 1997; Zhou,1999) for some results of this type. To the best of our knowledge, (Wernui et al., 2007) is the only published work on this topic for equation (1). Therefore, our main goal of this work is to obtain new estimates of the distance between adjacent zeros of all solutions of Eq.(1) under suitable criteria. We extended new techniques developed by (El-Morshedy, 2011) for the delay equation

$$
x^{\prime}(t)+P(t) x(t-\tau)=0,
$$

to the neutral equation (1) using new ideas from (Wernui 
etal., 2007). Our work is concluded with some illustrative examples. Throughout this work; $d_{s}(x)$ denotes the least upper bound of the distances between adjacent zeros of any solution $x(t)$ of Eq.(1) on $[s, \infty)$.

\section{PRELIMINARIES}

Let $d=\zeta_{0}\left(\frac{1}{p+1}\right)^{\frac{\sigma+\tau}{\tau}}$ and the sequences $\left\{a_{n}\right\}$ and $\left\{b_{m}\right\}$ be defined by

$$
a_{1}=\frac{p}{(p+1)^{2}}, \quad a_{n+1}=\frac{\frac{p}{(p+1)^{2}}}{1-a_{n}(1+d)}, n=1,2, \ldots
$$

and

$$
b_{1}=1+d, \quad b_{m+1}=\frac{1+d}{1-\frac{p}{(p+1)^{2}} b_{m}}, \quad m=1,2, \ldots
$$

These sequences are due to (Wernui et al., 2007). They showed that the number $\Delta=1-\frac{4(1+d) p}{(p+1)^{2}}$ governs some basic properties of $\left\{a_{n}\right\}$ and $\left\{b_{m}\right\}$ as in the following result which is derived from ([Lemma 1] Wernui et al., 2007) and [Rrmark 1].

Lemma 1 The sequences $\left\{a_{n}\right\}$ and $\left\{b_{m}\right\}$ converge if and only if $\Delta \geq 0$. Moreover; if $\Delta \geq 0$ then $\lim _{n \rightarrow \infty} a_{n}=$ $\frac{1-\sqrt{\Delta}}{2(1+d)}, \lim _{n \rightarrow \infty} b_{m}=\frac{1-\sqrt{\Delta}}{2 p}(1+p)^{2}$ and there exists $a$ positive integer $N_{1}$ such that

$$
\frac{1}{b_{m}}<\frac{p}{p+1} \quad \text { for } p>1, m \geq N_{1} .
$$

Next, for any solution $x(t)$ of Eq.(1), we define another related functions $y(t)$ and $z(t)$ as follows

$x(t)=y(t) e^{\beta t} \quad$ where $\beta=\frac{1}{\tau} \ln (p+1)\left(\right.$ or $\left.e^{\beta \tau}=p+1\right)$,

and

$$
z(t)=\int_{t-\tau}^{t} y(s) d s
$$

Lemma 2 ([lemma 2] Wernui etal., 2007) Let $\Delta \geq 0$ and $x(t)$ be a solution of Eq.(1) on $\left[t_{0}, \infty\right)$. For some $T_{1} \geq t_{0}+2 \tau$, if there exist a positive integer $N \geq 2$ and $T_{2} \geq T_{1}+N \tau+\sigma+\lambda$ such that $x(t)>0$ on $\left[T_{1}, T_{2}\right]$, then

$$
\frac{z(t)}{z(t-\tau)}>a_{n}, \quad \text { for } t \in\left[T_{1}+\tau+\sigma+\lambda, T_{2}-n \tau\right],
$$

for some $n \leq N-1$.

Lemma 3 ([lemma 3] Wernui etal., 2007) Let $\Delta \geq 0$ and $x(t)$ be a solution of Eq.(1) on $\left[t_{0}, \infty\right)$. For some $T_{1} \geq t_{0}+2 \tau$, if there exist a positive integer $N \geq 2$ and $T_{2} \geq T_{1}+N \tau+\sigma+\lambda$ such that $x(t)>0$ on $\left[T_{1}, T_{2}\right]$, then

$$
\frac{z(t)}{z(t-\tau)}<\frac{1}{b_{m}}, \quad \text { for } t \in\left[T_{1}+(m+1) \tau+\sigma+\lambda, T_{2}\right],
$$

Consider a sequence $\left\{\tilde{\mathrm{b}}_{m}\right\}$ defined as follows

$$
\tilde{\mathrm{b}}_{1}=1, \quad \tilde{\mathrm{b}}_{m+1}=\frac{1+d}{1-\frac{p}{(p+1)^{2}} \tilde{\mathrm{b}}_{m}}, \quad m=1,2, \ldots
$$

This sequence differs with $\left\{b_{m}\right\}$ in the initial term and both have the same properties explained in Lemma 1 when $\Delta \geq 0$. That is $\lim _{n \rightarrow \infty} \tilde{\mathrm{b}}_{m}=\frac{1-\sqrt{\Delta}}{2 p}(1+p)^{2}$ and there exists a positive integer $N_{2}$ such that

$$
\frac{1}{\tilde{\mathrm{b}}_{m}}<\frac{p}{p+1} \quad \text { for } p>1, m \geq N_{2} .
$$

The use of $\left\{\tilde{\mathrm{b}}_{m}\right\}$ enables us to prove the following version of Lemma 3.

Lemma 4 Let $\Delta \geq 0$ and $x(t)$ be a solution of Eq.(1) on $\left[t_{0}, \infty\right)$. If there exist a positive integer $N \geq 2, T_{1} \geq$ $t_{0}+2 \tau$ and $T_{2} \geq T_{1}+(N-1) \tau+2 \lambda$ such that $x(t)>0$ on $\left[T_{1}, T_{2}\right]$, then

$$
\frac{z(t)}{z(t-\tau)}<\frac{1}{\tilde{b}_{m}}, \quad \text { for } t \in\left[T_{1}+m \tau+2 \lambda, T_{2}\right],
$$

for some $m \leq N-1$.

Proof. If $x(t)>0$ on $\left[T_{1}, T_{2}\right]$ for $T_{1} \geq t_{0}+2 \tau$, it follows from (4) that $y(t)>0$, for $t \in\left[T_{1}, T_{2}\right]$ and hence (5) yields $z(t)>0$ on $\left[T_{1}+\tau, T_{2}\right]$. Integrating (1) from $t-\tau$ to $t$, we get

$$
\begin{gathered}
x(t)-(p+1) x(t-\tau)+p x(t-2 \tau) \\
+\int_{t-\tau}^{t} Q(s) x(s-\sigma) d s=0 .
\end{gathered}
$$

Using (4), we have

$$
\begin{gathered}
y(t)-y(t-\tau)+\frac{p}{(p+1)^{2}} y(t-2 \tau) \\
+e^{-\beta t} \int_{t-\tau}^{t} Q(s) y(s-\sigma) e^{\beta(s-\sigma)} d s=0 .
\end{gathered}
$$

Since $z^{\prime}(t)=y(t)-y(t-\tau)$, then (9) yields

$$
\begin{gathered}
z^{\prime}(t)=-\frac{p}{(p+1)^{2}} y(t-2 \tau) \\
-e^{-\beta t} \int_{t-\tau}^{t} Q(s) y(s-\sigma) e^{\beta(s-\sigma)} d s,
\end{gathered}
$$

which implies

$$
z^{\prime}(t)<0 \text {, for } t \in\left[T_{1}+\tau+\lambda, T_{2}\right] .
$$


Integrating (9) from $t-\tau$ to $t$,

$$
\begin{gathered}
z(t)-z(t-\tau)+\frac{p}{(p+1)^{2}} z(t-2 \tau) \\
+\int_{t-\tau}^{t} e^{-\beta s} d s \int_{s-\tau}^{s} Q(u) y(u-\sigma) e^{\beta(u-\sigma)} d u=0 .
\end{gathered}
$$

That is,

$$
\begin{gathered}
z(t)-z(t-\tau)+\frac{p}{(p+1)^{2}} z(t-2 \tau) \\
+\int_{t-\tau}^{t} Q_{*}(s) e^{-\beta(\tau+\sigma)} z(s-\sigma) d s \leq 0,
\end{gathered}
$$

for $t \in\left[T_{1}+2 \tau+\sigma, T_{2}\right]$. Hence

$$
\frac{z(t)}{z(t-\tau)}<1=\frac{1}{\tilde{\mathrm{b}}_{1}}, \quad \text { for } t \in\left[T_{1}+2 \tau+\lambda, T_{2}\right] .
$$

This inequality implies that

$$
z(t-2 \tau)>\tilde{\mathrm{b}}_{1} z(t-\tau)
$$

for $t \in\left[T_{1}+3 \tau+\lambda, T_{2}\right]$. Also (10) yields

$$
(1+d) z(t)-z(t-\tau)+\frac{p}{(p+1)^{2}} z(t-2 \tau)<0,
$$

for $t \in\left[T_{1}+2 \tau+\sigma+\lambda, T_{2}\right]$. Now, combining this inequality with (11), it follows that

$$
(1+d) z(t)-\left(1-\frac{p}{(p+1)^{2}} \tilde{\mathrm{b}}_{1}\right) z(t-\tau)<0,
$$

or

$$
(1+d) z(t)<\left(1-\frac{p}{(p+1)^{2}} \tilde{\mathrm{b}}_{1}\right) z(t-\tau),
$$

for $t \in\left[T_{1}+2 \tau+2 \lambda, T_{2}\right]$. Rearranging,

$$
\frac{z(t)}{z(t-\tau)}<\frac{1-\frac{p}{(p+1)^{2}} \tilde{\mathrm{b}}_{1}}{1+d}=\frac{1}{\tilde{\mathrm{b}}_{2}}, t \in\left[T_{1}+2 \tau+2 \lambda, T_{2}\right] .
$$

Similarly, for $t \in\left[T_{1}+3 \tau+2 \lambda, T_{2}\right]$, one can see that

$$
\frac{z(t)}{z(t-\tau)}<\frac{1-\frac{p}{(p+1)^{2}} \tilde{\mathrm{b}}_{2}}{(1+d)}=\frac{1}{\tilde{\mathrm{b}}_{3}}
$$

Repeating this argument till $m \leq L-1$, we obtain

$$
\frac{z(t)}{z(t-\tau)}<\frac{1}{\tilde{\mathrm{b}}_{m}}, \quad \text { for } t \in\left[T_{1}+m \tau+2 \lambda, T_{2}\right] .
$$

The proof is complete.
In the sequel, we consider the first order delay differential inequality

$$
x^{\prime}(t)-P(t) x(t+r) \geq 0,
$$

where $P \in\left(\left[t_{0}, \infty\right),[0, \infty)\right), r>0$ and

$$
\int_{t}^{t+r} P(s) d s \geq \rho, \quad t \geq t_{0} .
$$

for some constant $\rho>0$. We find some interesting results about the positivity of certain solution $x$ of (12) on some bounded intervals. For an easy reference, a sequence $\left\{A_{n}(t)\right\}$ is defined as follows

$$
\begin{aligned}
A_{0}(t)= & P(t), \quad t \geq t_{0} \\
A_{n}(t)= & A_{n-1}(t) \int_{t}^{t+r} A_{n-1}(s) e^{\int_{t}^{s+r} A_{n-1}(u) d u} d s, \\
& \text { for } \quad t \geq t_{0} \text { and } n=1,2, \ldots
\end{aligned}
$$

Lemma 5 Let $n$ be a positive integer such that

$$
\int_{t}^{t+r} A_{n}(s) d s \geq 1, \quad \text { for all } t \geq t_{0} .
$$

If $x(t)$ is a nondecreasing function on $\left[T_{1}, T_{2}+\delta\right]$ which satisfies (12) on $\left[T_{1}, T_{2}\right]$, then $x(t)$ cannot be positive on $\left[T_{1}, T_{2}\right]$, where $T_{2}>T_{1}+(3 n+1) r-\delta, T_{1} \geq t_{0}$ and $|\delta| \leq r$.

Proof. For the sake of contradiction, suppose that $x(t)$ is positive on $\left[T_{1}, T_{2}\right]$. Integrating (12) from $t$ to $t+r$, we obtain

$$
x(t+r)-x(t)-\int_{t}^{t+r} P(s) x(s+r) d s \geq 0,
$$

for $t \in\left[T_{1}, T_{2}-r\right]$. Using this inequality and (12), we obtain

$$
x^{\prime}(t)-P(t) x(t)-P(t) \int_{t}^{t+r} P(s) x(s+r) d s \geq 0,
$$

for $t \in\left[T_{1}, T_{2}-r\right]$. Let $y_{1}(t):=e^{-\int_{t_{0}}^{t} P(s) d s} x(t)$. Then $y_{1}(t)>0$ for $t \in\left[T_{1}, T_{2}\right]$ and the above inequality yields

$$
y_{1}^{\prime}(t)-P(t) \int_{t}^{t+r} P(s) e^{\int_{t}^{s+r} P(s) d s} y_{1}(s+r) d s \geq 0,
$$

for $t \in\left[T_{1}, T_{2}-r\right]$. Since $x(t)$ is nondecreasing on $\left[T_{1}, T_{2}+\delta\right]$, it follows that

$$
\begin{aligned}
y_{1}^{\prime}(t) & =\left(x^{\prime}(t)-P(t) x(t)\right) e^{-\int_{t_{0}}^{t} P(s) d s} \\
& \geq\left(x^{\prime}(t)-P(t) x(t+r)\right) e^{-\int_{t_{0}}^{t} P(s) d s} \\
& \geq 0, \quad \text { for } t \in\left[T_{1}, T_{2}+\delta-r\right] .
\end{aligned}
$$

Thus (14) leads to

$$
y_{1}^{\prime}(t)-A_{1}(t) y_{1}(t+r) \geq 0, \quad \text { for } t \in\left[T_{1}, T_{2}+\delta-3 r\right],
$$


which has the same form of (12) but with different coefficient. So using similar arguments as those applied for (12), we obtain

$$
y_{1}^{\prime}(t)-A_{1}(t) y_{1}(t)-A_{1}(t) \int_{t}^{t+r} A_{1}(s) y_{1}(s+r) d s \geq 0,
$$

for $t \in\left[T_{1}, T_{2}+\delta-4 r\right]$. Set $y_{2}(t):=e^{-\int_{t_{0}}^{t} A_{1}(s) d s} y_{1}(t)$. Then

$$
y_{2}^{\prime}(t)-A_{1}(t) \int_{t}^{t+r} A_{1}(s) e^{\int_{t}^{s+r} A_{1}(s) d s} y_{2}(s+r) d s \geq 0,
$$

for $t \in\left[T_{1}, T_{2}+\delta-4 r\right]$, and thus $y_{2}^{\prime}(t) \geq 0$ for $t \in$ $\left[T_{1}, T_{2}+\delta-4 r\right]$. Hence

$$
y_{2}^{\prime}(t)-A_{2}(t) y_{2}(t+r) \geq 0, \quad \text { for } t \in\left[T_{1}, T_{2}+\delta-6 r\right] .
$$

So, an induction yields

$$
y_{n}^{\prime}(t)-A_{n}(t) y_{n}(t+r) \geq 0, \quad \text { for } t \in\left[T_{1}, T_{2}+\delta-3 n r\right],
$$

where $y_{n}^{\prime}(t) \geq 0$ for $t \in\left[T_{1}, T_{2}+\delta-(3 n-2) r\right]$. Integrate (15) from $t$ to $t+r$, we obtain

$$
y_{n}(t+r)-y_{n}(t)-\int_{t}^{t+r} A_{n}(s) y_{n}(s+r) d s \geq 0,
$$

for $t \in\left[T_{1}, T_{2}+\delta-(3 n+1) r\right]$.Therefore

$$
0<y_{n}(t) \leq\left[1-\int_{t}^{t+r} A_{n}(s) d s\right] y_{n}(t+r) \leq 0,
$$

for $t \in\left[T_{1}, T_{2}+\delta-(3 n+1) r\right]$, which is impossible. The proof is complete.

In the next lemma, we give an interesting result that determine lower bound for the ratio $\frac{x(t+\tau)}{x(t)}$ by making use of the sequence $\left\{f_{n}(\rho)\right\}$ defined by (Xianhua and Jianshe, 1999), for $0<\rho<1$, as follows

$$
\begin{gathered}
f_{0}(\rho)=1, \quad f_{1}(\rho)=\frac{1}{1-\rho}, \\
f_{n+2}(\rho)=\frac{f_{n}(\rho)}{f_{n}(\rho)+1-e^{\rho f_{n}(\rho)}}, n=0,1, \ldots
\end{gathered}
$$

where $\rho$ is defined by (13). According to (Xianhua and Jianshe, 1999), the sequence $f_{n+2}(\rho)$ could be positive, negative as some $n \geq 0$ or its denominator $\left(f_{n}(\rho)+1-\right.$ $\left.e^{\rho f_{n}(\rho)}\right)$ is zero. In the last case we say that $f_{n+2}(\rho)=\infty$.

Lemma 6 Assume that (13) holds for $0<\rho<1$ and there exist $T_{1} \geq t_{0},|\delta| \leq r, T \geq T_{1}+(n+1) r-\delta$ and a function $x(t)$ satisfying inequality (12) on $\left[T_{1}, T\right]$ with $x^{\prime}(t) \geq 0$ for $t \in\left[T_{1}, T+\delta\right]$. If $x(t)$ is positive on $\left[T_{1}, T\right]$, then

$$
\frac{x(t+r)}{x(t)} \geq f_{n}(\rho)>0 \text { for } t \in\left[T_{1}, T-(n+1) r+\delta\right],
$$

for some integer $n \geq 0$, where $f_{n}(\rho)$ is defined by (16).
Proof. Since $x(t)$ is nondecreasing on $\left[T_{1}, T+\delta\right]$. It follows that

$$
\frac{x(t+r)}{x(t)} \geq 1=f_{0}(\rho) \quad \text { for } t \in\left[T_{1}, T-r+\delta\right] .
$$

Integrating inequality (12) from $t$ to $t+r$, we have

$$
x(t+r)-x(t) \geq \int_{t}^{t+r} P(s) x(s+r) d s \geq \rho x(t+r),
$$

for $t \in\left[T_{1}, T-2 r+\delta\right]$. That is,

$$
\frac{x(t+r)}{x(t)} \geq \frac{1}{1-\rho}=f_{1}(\rho)>0 \text { for } t \in\left[T_{1}, T-2 r+\delta\right] .
$$

Now, when $t \in\left[T_{1}, T-3 r+\delta\right]$, integrate inequality (12) from $t$ to $t+r$,

$$
x(t+r) \geq x(t)+\int_{t}^{t+r} P(s) x(s+r) d s .
$$

Dividing both sides of (12) by $x(t)$ and integrating from $t+r$ to $s+r$, we obtain

$$
\begin{aligned}
\frac{x(s+r)}{x(t+r)} & \geq \exp \left(\int_{t+r}^{s+r} P(u) \frac{x(u+r)}{x(u)} d u\right) \\
& \geq \exp \left(f_{0}(\rho) \int_{t+r}^{s+r} P(u) d u\right) .
\end{aligned}
$$

Using this inequality and (18), we get

$$
\begin{aligned}
x(t+r) \geq & x(t)+x(t+r) \int_{t}^{t+r} P(s) \frac{x(s+r)}{x(t+r)} d s \\
\geq & x(t)+x(t+r) \int_{t}^{t+r} P(s) \\
& \exp \left(f_{0}(\rho) \int_{t+r}^{s+r} P(u) d u\right) d s \\
= & x(t)+x(t+r) \int_{t}^{t+r} P(s) \exp \\
& {\left[f_{0}(\rho)\left(\int_{s}^{s+r} P(u) d u-\int_{s}^{t+r} P(u) d u\right)\right] d s } \\
\geq & x(t)+x(t+r) e^{\rho f_{0}(\rho)} \int_{t}^{t+r} P(s) \exp \\
& \left(-f_{0}(\rho) \int_{s}^{t+r} P(u) d u\right) d s \\
= & x(t)+\frac{x(t+r) e^{\rho f_{0}(\rho)}}{f_{0}(\rho)} \\
& -\frac{x(t+r) e^{\rho f_{0}(\rho)} e x p\left(-f_{0}(\rho) \int_{t}^{t+r} P(u) d u\right.}{f_{0}(\rho)} \\
= & x(t)+\frac{x(t+r)\left(e^{\rho f_{0}(\rho)}-1\right)}{f_{0}(\rho)} .
\end{aligned}
$$


So

$$
\frac{x(t+r)}{x(t)} \geq \frac{f_{0}(\rho)}{f_{0}(\rho)+1-e^{\rho f_{0}(\rho)}}=f_{2}(\rho)>0,
$$

for $t \in\left[T_{1}, T-3 r+\delta\right]$. Repeating the above steps yields

$$
\frac{x(t+r)}{x(t)} \geq f_{n}(\rho)>0 \text { for } t \in\left[T_{1}, T-(n+1) r+\delta\right] .
$$

The proof of Lemma 6 is complete.

Next, we need a sequence $\left\{q_{n}(s)\right\}_{n \geq 1}$ defined for $s \in$ $(t, t+\tau)$ as follows

$$
\begin{aligned}
q_{1}(s) & :=P(s) \\
q_{n+1}(s) & :=P(s+n r) \int_{s}^{t+r} P_{n}(u) d u, t \geq t_{0},
\end{aligned}
$$

and we consider that $\sum_{k=a}^{b} L_{k}=1$ for any sequence $\left\{L_{n}\right\}$ as long as $b<a$.

Lemma 7 Let $n^{*}$ and $n^{* *}$ be two positive integers such that $n^{* *}=\min \left\{l: f_{l+1}(\rho)<0\right.$ or $\left.f_{l+1}(\rho)=\infty\right\}$ and

$$
\sum_{k=1}^{n^{*}}\left(\prod_{i=2}^{k} f_{n^{*}+2-i}(\rho)\right) \int_{t}^{t+r} q_{k}(s) d s \geq 1, \quad t \geq t_{0},
$$

for $\rho \in(0,1)$. Further; assume that $x(t)$ is nondecreasing on $\left[T_{1}, T_{2}+\delta\right]$, where $T_{1} \geq t_{0}$ and $|\delta| \leq r$. If $x(t)$ satisfies (12) on $\left[T_{1}, T_{2}\right]$, then $x(t)$ cannot be positive on $\left[T_{1}, T_{2}\right]$, where $T_{2}>T_{1}+(n+2) r-\delta$ and $n=\min \left\{n^{*}, n^{* *}\right\}$.

Proof. Suppose, for the sake of contradiction, that $x(t)$ is positive on $\left[T_{1}, T_{2}\right]$. If $n=n^{* *}$, then Lemma 6 implies a contradiction. Thus, assume that $n=n^{*}$ and integrate (12) from $t$ to $t+r$, we have

$$
x(t+r)-x(t)-\int_{t}^{t+r} P(s) x(s+r) d s \geq 0,
$$

for $t \in\left[T_{1}, T_{2}-r\right]$. Using integration by parts, we obtain

$$
\begin{aligned}
& \int_{t}^{t+r} P(s) x(s+r) d s \\
& =\int_{t}^{t+r} x(s+r) d\left(-\int_{s}^{t+r} P(u) d u\right) \geq x(t+r) \\
& \int_{t}^{t+r} q_{1}(s) d s+\int_{t}^{t+r} q_{2}(s) x(s+2 r) \mathrm{ds} \\
& \geq\left(\int_{t}^{t+r} q_{1}(s) d s\right) x(t+r)+\left(\int_{t}^{t+r} q_{2}(s) d s\right) x(t+2 r) \\
& \int_{t}^{t+r} q_{3}(s) x(s+3 r) d s, \quad \text { for } t \in\left[T_{1}, T_{2}-3 r\right] .
\end{aligned}
$$

Continuing the above arguments $n$ times, we find

$$
\int_{t}^{t+r} P(s) x(s+r) d s \geq \sum_{k=1}^{n}\left(\int_{t}^{t+r} q_{k}(s) d s\right) x(t+k r)
$$

$+\int_{t}^{t+r} q_{n+1}(s) x(s+(n+1) r) d s, t \in\left[T_{1}, T_{2}-(n+1) r\right]$.

Since $x(s+(n+1) r)>0$ for $s \in\left[T_{1}, T_{2}+\delta-(n+1) r\right]$, then

$$
\int_{t}^{t+r} P(s) x(s+r) d s \geq \sum_{k=1}^{n}\left(\int_{t}^{t+r} q_{k}(s) d s\right) x(t+k r),
$$

for $t \in\left[T_{1}, T_{2}+\delta-(n+2) r\right]$. On the other hand, for $t \in\left[T_{1}, T_{2}+\delta-(n+2) r\right]$, we have $x(t)>0$ and

$t+(i-1) r \in\left[T_{1}, T_{2}+\delta-(n+3-i) r\right], \quad i=2,3, \ldots, n$.

So, when $t \in\left[T_{1}, T_{2}+\delta-(n+2) r\right]$, Lemma 6 implies that

$$
\frac{x(t+i r)}{x(t+(i-1) r)} \geq f_{n+2-i}(\rho), \quad i=2,3, \ldots, n .
$$

Therefore,

$$
\begin{aligned}
x(t+k r) & =\left(\prod_{i=2}^{k} \frac{x(t+i r)}{x(t+(i-1) r)}\right) x(t+r) \\
& \geq\left(\prod_{i=2}^{k} f_{n+2-i}(\rho)\right) x(t+r), k=1,2, \ldots, n .
\end{aligned}
$$

Using the above inequality and (20), we get

$$
\begin{array}{r}
\int_{t}^{t+r} P(s) \quad x(s+r) d s \geq \sum_{k=1}^{n}\left(\int_{t}^{t+r} q_{k}(s) d s\right) x(t+k r) \\
\geq \sum_{k=1}^{n}\left(\prod_{i=2}^{k} f_{n+2-i}(\rho) \int_{t}^{t+r} q_{k}(s) d s\right) x(t+r),
\end{array}
$$

for $t \in\left[T_{1}, T_{2}+\delta-(n+2) r\right]$. Thus (19) leads to

$x(t) \leq\left[1-\sum_{k=1}^{n} \prod_{i=2}^{k} f_{n+2-i}(\rho) \int_{t}^{t+r} q_{k}(s) d s\right] x(t+r) \leq 0$.

This contradiction completes the proof.

\section{MAIN RESULTS}

In the following results, we restrict ourselves to the cases when $\Delta \geq 0$ and $\tau>\sigma$. It will be assumed that

$$
\int_{t}^{t+\tau-\sigma} Q_{*}(s) d s \geq \zeta_{1}, \quad t \geq t_{1}
$$

for some positive constant $\zeta_{1}$ and $t_{1} \geq t_{0}+2 \tau$.

In view of Lemma 1 we have $\lim _{n \rightarrow \infty} a_{n}=\frac{1-\sqrt{\Delta}}{2(1+d)}$. So the sequence $\left\{\eta_{n}\right\}$ where $\eta_{n}=\frac{\zeta_{1}}{p-a_{n}(p+1)}, n>0$, converges to a number $\eta=\frac{\zeta_{1}}{p-a^{0}(p+1)}$. We define a sequence 
$\left\{A_{n, j}(t)\right\}$ for any positive integer $j$ by

$$
\begin{aligned}
A_{0, j}(t)= & \frac{Q_{*}(t)}{p-a_{j}(p+1)}, \quad t \geq t_{1} \\
A_{n, j}(t)= & A_{n-1, j}(t) \int_{t}^{t+\tau-\sigma} A_{n-1, j}(s) \exp ( \\
& \left.\int_{t}^{s+\tau-\sigma} A_{n-1, j}(u) d u\right) d s, t \geq t_{1}, n=1,2, \ldots
\end{aligned}
$$

Theorem 1 Assume that (21) holds. Let $n$ be a positive integer such that

$$
\int_{t}^{t+\tau-\sigma} A_{n, j^{*}}(s) d s \geq 1, \quad t \geq t_{1} .
$$

Then Eq.(1) is oscillatory and $d_{t_{1}}(x) \leq\left(m^{\prime}+j^{*}+3\right) \tau+$ $3 n(\tau-\sigma)$, where

$$
j^{*}=\min \left\{j \mid \eta_{j}>0\right\},
$$

and

$$
m^{\prime}=\min _{m \geq 1}\left\{m \mid \frac{1}{b_{m}}<\frac{p}{p+1}\right\}
$$

Proof. Let $x(t)$ be a solution of Eq.(1) with $x(t)>0$ on $\left[T_{1}, T_{2}\right]$ where $T_{2}>T_{1}+\left(m^{\prime}+2\right) \tau+j^{*} \tau+\sigma+(3 n+$ 1) $(\tau-\sigma), T_{1} \geq t_{1}$. In view of (4), Eq.(1) implies that

$$
\begin{gathered}
{\left[y(t)-\frac{p}{p+1} y(t-\tau)\right]^{\prime}+\beta\left[y(t)-\frac{p}{p+1} y(t-\tau)\right]} \\
+Q(t) e^{-\beta \sigma} y(t-\sigma)=0 .
\end{gathered}
$$

Integrating from $t-\tau$ to $t$, we get

$$
\begin{gathered}
{\left[z(t)-\frac{p}{p+1} z(t-\tau)\right]^{\prime}+\beta\left[z(t)-\frac{p}{p+1} z(t-\tau)\right]} \\
+e^{-\beta \sigma} \int_{t-\tau}^{t} Q(s) y(s-\sigma) d s=0
\end{gathered}
$$

which yields

$$
\begin{gathered}
{\left[z(t)-\frac{p}{p+1} z(t-\tau)\right]^{\prime}+\beta\left[z(t)-\frac{p}{p+1} z(t-\tau)\right]} \\
+e^{-\beta \sigma} Q_{*}(t) z(t-\sigma) \leq 0 \text { for } t \in\left[T_{1}+\tau+\sigma, T_{2}\right] .
\end{gathered}
$$

Since $\Delta \geq 0$ and $p>1$, it follows from (3) and Lemma 3 that

$$
\frac{z(t)}{z(t-\tau)}<\frac{1}{b_{m}}<\frac{1}{b_{m^{\prime}}}<\frac{p}{p+1},
$$

for any $m \geq m^{\prime}$ where $m^{\prime}$ is defined by (24) and $t \in$ $\left[T_{1}+\left(m^{\prime}+2\right) \tau+\sigma, T_{2}\right]$. Set

$u(t)=z(t)-\frac{p}{p+1} z(t-\tau), \quad t \in\left[T_{1}+\left(m^{\prime}+2\right) \tau+\sigma, T_{2}\right]$.

Then (26) gives

$$
u(t)<0, \quad \text { for } t \in\left[T_{1}+\left(m^{\prime}+2\right) \tau+\sigma, T_{2}\right] .
$$

Moreover; Lemma 2 yields

$$
\begin{aligned}
u(t) & =z(t)-\frac{p}{p+1} z(t-\tau) \\
& =\left[\frac{z(t)}{z(t-\tau)}-\frac{p}{p+1}\right] z(t-\tau) \\
& >\left[a_{j}-\frac{p}{p+1}\right] z(t-\tau),
\end{aligned}
$$

for $t \in\left[T_{1}+2 \tau+\sigma, T_{2}-j \tau\right]$ and any positive integer $j$. Thus

$$
u(t+\tau-\sigma)>\left[a_{j}-\frac{p}{p+1}\right] z(t-\sigma),
$$

for $t \in\left[T_{1}+2 \tau+\sigma, T_{2}-(j+1) \tau+\sigma\right]$. Substituting (27) and (28) into (25),

$$
u^{\prime}(t)+\beta u(t)-\frac{Q_{*}(t) e^{-\beta \sigma}}{\frac{p}{p+1}-a_{j}} u(t+\tau-\sigma)<0,
$$

for $t \in\left[T_{1}+2 \tau+\sigma, T_{2}-(j+1) \tau+\sigma\right]$. Put $w(t)=$ $-e^{\beta t} u(t)$, for $t \in\left[T_{1}+\left(m^{\prime}+2\right) \tau+\sigma, T_{2}\right]$ and take $j=j^{*}$, $T=T_{1}+\left(m^{\prime}+2\right) \tau+\sigma$, then

$$
w(t)>0, \quad \text { for } t \in[T, T+3 n(\tau-\sigma)],
$$

and inequality (25) leads to

$$
\begin{aligned}
w^{\prime}(t) & =-\left(u^{\prime}(t)+\beta u(t)\right) e^{\beta t} \\
& \geq e^{\beta(t-\sigma)} Q_{*}(t) z(t-\sigma)>0, t \in\left[T-m^{\prime} \tau, T_{2}\right] .
\end{aligned}
$$

Hence $\left.w^{\prime} t\right)>0$ on $[T, T+(3 n+1)(\tau-\sigma)]$ and $(29)$ is transformed to the form

$$
w^{\prime}(t)-\frac{Q_{*}(t)}{p-a_{j^{*}}(p+1)} w(t+\tau-\sigma)>0,
$$

for $t \in[T, T+3 n(\tau-\sigma)]$. Using Lemma 5 , with $\delta=\tau-\sigma$ and $A_{n}$ is replaced by $A_{n, j}$, we conclude that $w(t)$ cannot be positive on $[T, T+3 n(\tau-\sigma)]$. This contradiction completes the proof.

Corollary 1 Assume that (21) holds. Let $\left\{\alpha_{n, j^{*}}\right\}_{n \geq 1}$ be a sequence defined by

$$
\alpha_{n, j^{*}}=\alpha_{n-1, j^{*}}\left(e^{2 \alpha_{n-1, j^{*}}}-e^{\alpha_{n-1, j^{*}}}\right), \quad \alpha_{0, j^{*}}=\eta_{j^{*}} .
$$

If there exists a positive integer $n_{0}$ satisfying $\alpha_{n_{0}, j^{*}} \geq 1$, then Eq.(1) is oscillatory and $d_{t_{1}}(x) \leq\left(m^{\prime}+3+j^{*}\right) \tau+$ $3 n_{0}(\tau-\sigma)$, where $j^{*}$ is defined by (23) and $m^{\prime}$ is defined by (24). 
Proof. From (21), it follows that

$$
\int_{t}^{t+\tau-\sigma} A_{0, j^{*}}(s) d s \geq \alpha_{0, j^{*}}=\eta_{j^{*}}
$$

Thus

$$
\begin{aligned}
& \int_{t}^{t+\tau-\sigma} A_{1, j^{*}}(s) d s \\
= & \int_{t}^{t+\tau-\sigma} A_{0, j^{*}}(s) \int_{s}^{s+\tau-\sigma} A_{0, j^{*}}(u) \\
& e^{\int_{s}^{u+\tau-\sigma} A_{0, j^{*}}(v) d v} d u d s \\
= & \int_{t}^{t+\tau-\sigma} A_{0, j^{*}}(s) \int_{s}^{s+\tau-\sigma} A_{0, j^{*}}(u) e^{\int_{s}^{u} A_{0, j^{*}}(v) d v} \\
& e^{\int_{u}^{u+\tau-\sigma} A_{0, j^{*}}(v) d v} d u d s \\
\geq & e^{\alpha_{0, j^{*}}} \int_{t}^{t+\tau-\sigma} A_{0, j^{*}}(s) \\
& \left(\int_{s}^{s+\tau-\sigma} A_{0, j^{*}}(u) e^{\int_{s}^{u} A_{0, j^{*}}(v) d v} d u\right) d s \\
= & e^{\alpha_{0, j^{*}}} \int_{t}^{t+\tau-\sigma} A_{0, j^{*}}(s)\left(e^{\int_{s}^{s+\tau-\sigma} A_{0, j^{*}}(v) d v}-1\right) d s \\
\geq & e^{\alpha_{0, j^{*}}}\left(e^{\alpha_{0, j^{*}}}-1\right) \int_{t}^{t+\tau-\sigma} A_{0, j^{*}}(s) d s \geq \alpha_{1, j^{*}} .
\end{aligned}
$$

By induction, it follows that

$$
\int_{t}^{t+\tau-\sigma} A_{n, j^{*}}(s) d s \geq \alpha_{n, j^{*}}, \quad \text { for } n=0,1, \ldots
$$

Thus, when $\alpha_{n_{0}, j^{*}} \geq 1$, then (22) holds for $n=n_{0}$. Therefore, the proof can be completed by applying Theorem 1 .

In the following result, we consider the sequence $\left\{q_{n, j}(s)\right\}$ defined by

$$
\begin{aligned}
q_{1, j}(s) & =\frac{Q_{*}(s)}{p-a_{j}(p+1)}, \\
q_{n+1, j}(s) & =q_{1, j}(s+n(\tau-\sigma)) \int_{s}^{t+\tau-\sigma} q_{n, j}(u) d u,
\end{aligned}
$$

for $t \geq t_{1}$ and $n \geq 1$.

Theorem 2 Assume that (21) holds. If $n^{*}, n^{* *}$ are two positive integers such that $n^{* *}=\min \left\{l: f_{l+1}\left(\eta_{j^{*}}\right)<0\right.$ or $\left.f_{l+1}\left(\eta_{j^{*}}\right)=\infty\right\}$ and

$$
\sum_{k=1}^{n^{*}}\left(\prod_{i=2}^{k} f_{n^{*}+2-i}\left(\eta_{j^{*}}\right)\right) \int_{t}^{t+\tau-\sigma} q_{k, j^{*}}(s) d s \geq 1,
$$

for $t \geq t_{1}$ and $\eta_{j^{*}} \in(0,1)$, then Eq.(1) is oscillatory and $d_{t_{1}}(x) \leq\left(m^{\prime}+3+j^{*}\right) \tau+(n+1)(\tau-\sigma)$ for any solution $x(t)$ of (1), where $j^{*}, m^{\prime}$ are defined by (23), (24) respectively and $n=\min \left\{n^{*}, n^{* *}\right\}$.
Proof. Assume, for the sake of contradiction, that $x(t)>0$ for $t \in\left[T_{1}, T_{1}+\left(m^{\prime}+2\right) \tau+\sigma+j^{*} \tau+(n+\right.$ $2)(\tau-\sigma)]$. We take $n=n^{*}$ since otherwise a contradiction appears. Proceeding as in Theorem 1, we obtain the inequality

$$
w^{\prime}(t)-\frac{Q_{*}(t)}{p-a_{j^{*}}(p+1)} w(t+\tau-\sigma)>0,
$$

for $t \in[T, T+(n+1)(\tau-\sigma)]$, and

$$
\left.w^{\prime} t\right)>0, \quad \text { for } t \in[T, T+(n+2)(\tau-\sigma)] .
$$

Moreover, (30) yields

$$
w(t)>0 \quad \text { for } t \in[T, T+(n+1)(\tau-\sigma)] .
$$

According to Lemma 7, with $\delta=\sigma-\tau$ and $q_{n}$ is replaced by $q_{n, j}$, we obtain that $w(t)$ cannot be positive on $[T, T+$ $(n+1)(\tau-\sigma)]$. This contradiction completes the proof.

Since $f_{2}\left(\eta_{j^{*}}\right)=\frac{1}{2-e^{\eta_{j^{*}}}}$ for $\eta_{j^{*}} \in(0,1)$, then Theorem 2 and definition of $n^{* *}$ lead to the particular result.

Corollary 2 Assume that (21) holds. If either $\ln 2 \leq$ $\eta_{j^{*}}<1$ or $0<\eta_{j^{*}}<\ln 2$ and

$$
\begin{aligned}
& \int_{t}^{t+\tau-\sigma} q_{1, j^{*}}(s) d s+f_{2}\left(\eta_{j^{*}}\right) \int_{t}^{t+\tau-\sigma} q_{1, j^{*}}(s+\tau-\sigma) \\
& \int_{s}^{t+\tau-\sigma} q_{1, j^{*}}(u) d u d s \geq 1, \quad t \geq t_{1},
\end{aligned}
$$

then Eq.(1) is oscillatory and $d_{t_{1}}(x) \leq\left(m^{\prime}+3+j^{*}\right) \tau+$ $3(\tau-\sigma)$, where $j^{*}, m^{\prime}$ are defined by (23), (24) respectively.

Corollary 3 Assume that (21) holds and

$$
\frac{\zeta_{1}}{p-a_{j}(p+1)} \geq \eta_{j^{*}} \quad \text { for } \eta_{j^{*}} \in(0,1) .
$$

If $n^{*}, n^{* *}$ are two positive integers such that $n^{* *}=$ $\min \left\{l: f_{l+1}\left(\eta_{j^{*}}\right)<0\right.$ or $\left.f_{l+1}\left(\eta_{j^{*}}\right)=\infty\right\}$ and

$$
\sum_{k=1}^{n^{*}} \prod_{i=2}^{k} f_{n^{*}-(i-2)}\left(\eta_{j^{*}}\right) \frac{\eta_{j^{*}}^{k}}{k !} \geq 1
$$

then Eq.(1) is oscillatory and $d_{t_{1}}(x) \leq\left(m^{\prime}+3+j^{*}\right) \tau+$ $(n+1)(\tau-\sigma)$, where $j^{*}, m^{\prime}$ are defined by (23), (24) respectively and $n=\min \left\{n^{*}, n^{* *}\right\}$.

Example 1 Consider the neutral differential equation

$[x(t)-5.6 x(t-3)]^{\prime}+\left(2.6+e^{-5 t}\right) x(t-2)=0, \quad t>0$, 
which has the form (1) with $p=5.6, Q(t)=2.6$ $+e^{-5 t}, \tau=3$ and $\sigma=2$. For $t \geq t_{1}=6$, we have

$$
\begin{gathered}
\int_{t-\tau}^{t} Q_{*}(s) d s \geq 7.8=\zeta_{0}, \\
\int_{t}^{t+\tau-\sigma} Q_{*}(s) d s \geq 2.6=\zeta_{1}, \\
d=0.33588 \ldots, \text { and } \Delta>0 .
\end{gathered}
$$

So, it follows

$$
\begin{aligned}
& a_{1}=0.12855 \ldots, \quad b_{1}=1.33588 \ldots, \quad \eta_{1}=0.5471 \ldots, \\
& f_{1}\left(\eta_{1}\right)=2.2084 \ldots, \quad f_{2}\left(\eta_{1}\right)=3.6818 \ldots
\end{aligned}
$$

Clearly, $\frac{1}{b_{1}}<\frac{p}{p+1}, m^{\prime}=1, j^{*}=1$ and $\eta_{1}+f_{2}\left(\eta_{1}\right) \frac{\eta_{1}^{2}}{2}=$ 1.09. Applying Corollary 3 , it follows that (35) holds for $n=n^{*}=2$ and $d_{6}(x) \leq\left(m^{\prime}+j^{*}+3\right) \tau+(n+1)(\tau-\sigma)=$ 18. Theorem 3 in (Wenrui etal., 2007) can not give an estimation smaller than 18 .

All previous results of this section can be proved using $\left\{\tilde{\mathrm{b}}_{m}\right\}$ instead of $\left\{b_{m}\right\}$. The obtained results lead to better estimates in some situations. Next, we list those new results without proofs.

Theorem 3 Assume that (21) holds. Let $n$ be a positive integer such that (22) holds. Then Eq.(1) is oscillatory and $d_{t_{1}}(x) \leq\left(m^{\prime \prime}+j^{*}+2\right) \tau+(3 n+1)(\tau-\sigma)$, where $j^{*}$ is defined by (23) and

$$
m^{\prime \prime}=\min _{m \geq 2}\left\{m \mid \frac{1}{\tilde{b}_{m}}<\frac{p}{p+1}\right\} .
$$

Corollary 4 Assume that (21) holds. Let $\left\{\alpha_{n, j^{*}}\right\}$ be defined by (31). If there exists a positive integer $n_{0}$ satisfying $\alpha_{n_{0}, j^{*}} \geq 1$, then Eq.(1) is oscillatory and $d_{t_{1}}(x) \leq\left(m^{\prime \prime}+2+j^{*}\right) \tau+\left(3 n_{0}+1\right)(\tau-\sigma)$, where $j^{*}$ is defined by (23) and $m^{\prime \prime}$ is defined by (36).

Theorem 4 Assume that (21) holds. If $n^{*}, n^{* *}$ are two positive integers such that $n^{* *}=\min \left\{l: f_{l+1}\left(\eta_{j^{*}}\right)<0\right.$ or $\left.f_{l+1}\left(\eta_{j^{*}}\right)=\infty\right\}$ and (32) holds for $\eta_{j^{*}} \in(0,1)$, then Eq. (1) is oscillatory and $d_{t_{1}}(x) \leq\left(m^{\prime \prime}+2+j^{*}\right) \tau+(n+$ $2)(\tau-\sigma)$ for any solution $x(t)$ of $(1)$, where $j^{*}, m^{\prime \prime}$ are defined by (23), (36) respectively and $n=\min \left\{n^{*}, n^{* *}\right\}$.

Corollary 5 Assume that (21) holds. If either $\ln 2 \leq \eta_{j^{*}}<1$ or $0<\eta_{j^{*}}<\ln 2$ and (33) holds, then Eq. (1) is oscillatory and $d_{t_{1}}(x) \leq\left(m^{\prime \prime}+2+j^{*}\right) \tau+4(\tau-\sigma)$, where $j^{*}, m^{\prime \prime}$ are defined by (23), (36) respectively.

Corollary 6 Assume that (21) and (34) hold. If $n^{*}$, $n^{* *}$ are two positive integers such that $n^{* *}=\min \{l$ : $f_{l+1}\left(\eta_{j^{*}}\right)<0$ orf $\left.f_{l+1}\left(\eta_{j^{*}}\right)=\infty\right\}$ and (35) holds, then Eq. (1) is oscillatory and $d_{t_{1}}(x) \leq\left(m^{\prime \prime}+2+j^{*}\right) \tau+(n+$ $2)(\tau-\sigma)$, where $j^{*}, m^{\prime \prime}$ are defined by (23), (36) respectively and $n=\min \left\{n^{*}, n^{* *}\right\}$.
Example 2 Consider the neutral differential equation

$$
[x(t)-3 x(t-2)]^{\prime}+\left(1.2+\frac{2}{t}\right) x(t-1)=0, \quad t>0,
$$

Then $p=3, Q(t)=1.2+\frac{2}{t}, \tau=2, \sigma=1$ and for $t \geq t_{1}=4$, we have

$$
\begin{gathered}
\int_{t-\tau}^{t} Q_{*}(s) d s \geq 2.4=\zeta_{0}, \\
\int_{t}^{t+\tau-\sigma} Q_{*}(s) d s \geq 1.2=\zeta_{1}, \\
d=0.3 \ldots, \text { and } \Delta>0 .
\end{gathered}
$$

In addition, we note that

$$
\begin{aligned}
& a_{1}=0.1875 \ldots, \quad b_{1}=1.3, \quad b_{2}=1.719 \ldots, \quad \tilde{\mathrm{b}}_{1}=1, \\
& \tilde{\mathrm{b}}_{2}=1.6, \eta_{1}=0.5333 \ldots, \quad f_{1}\left(\eta_{1}\right)=2.1427 \ldots, \\
& f_{2}\left(\eta_{1}\right)=3.3846 \ldots
\end{aligned}
$$

Therefore, $m^{\prime}=m^{\prime \prime}=2, j^{*}=1$. Applying Corollary 6 , we find that $\eta_{1}+f_{2}\left(\eta_{1}\right) \frac{\eta_{1}^{2}}{2}>1$. Then $n=$ $n^{*}=2$ and $d_{4}(x) \leq\left(m^{\prime \prime}+j^{*}+2\right) \tau+(n+2)(\tau-$ $\sigma)=14$. One can see that Corollary 3 gives that $d_{4}(x) \leq\left(m^{\prime}+j^{*}+3\right) \tau+(n+1)(\tau-\sigma)=15$.

Remark 1 Two sets of results are obtained. One using $\left\{b_{m}\right\}$ and the other using the modified sequence $\left\{\tilde{b}_{m}\right\}$. In example 2, it was shown that Corollary 6 gives better estimates than Corollary 3. But if we apply Corollary 6 to example 1 , it is easy to see that $d_{6}(x) \leq 19$. So Corollary 3 leads to better estimates in this case. This emphasizes the importance of our new sequence $\left\{\tilde{b}_{m}\right\}$ and shows that these sets are independent of each other.

\section{Acknowledgements}

The authors would like to thank the referees for their valuable comments and suggestions.

\section{References}

Agarwal et al. (2012) Nonoscillation Theory of Functional Differential Equations with Applications. Springer.

Bainov, Mishev (1991) Oscillation Theory for Neutral Differential Equations with Delay. Adam Hilger.

Erbe etal. (1995) Oscillation Theory for Functional Differential Equations. Dekker, New York.

El-Morshedy HA (2011) On the distribution of zeros of solutions of first order delay differential equations. Nonlinear Anal. 74: $3353-3362$

Gopalsamy K (1992) Stability and Oscillation in Delay Differential Equations of Population Dynamics. Kluwer Academic Publishers, Dordrecht. 
Györi, Ladas (1991) Oscillation Theory of Delay Differential Equations with Applications. Clarendon Press, Oxford. Hale, Lunel (1993) Introduction to Functional Differential Equations. Springer- Verlag, New York.

Kolmanovskii, Myshkis (1999) Introduction to the Theory and Applications of Functional Differential Equations. Kluwer Academic Publishers, Netherlands.

Kolmanovskii, Nosov (1986) Stability of Functional Differential Equations. Academic Press, London.

Wenrui etal. (2007) The distribution of zeros of solutions for a class of neutral delay differential equations. Appl. Math. Comput. 186: 1137-1150.

Wu etal. (2007) Distribution of zeros of solutions of func- tional differential equations. Appl. Math. Comp. 193: 154-161.

Wu, Xu (2004) The distribution of zeros of solutions of neutral differential equations. Appl. Math. Comp. 156: 665-677.

Xianhua, Jianshe (1999) Distribution of zeros of solutions of first order delay differential equations. Appl. Math. J. Chinese Univ. Ser. B, 14: 375-380.

Yong, Zhicheng (1997) The distribution of zeros of solutions of neutral equations. Appl. Math. Mechanics (English Ed.) 18: 1197-1204.

Zhou Y (1999) The distribution of zeros of solutions of neutral differential equations. Hiroshima. Math. J. 29: 361-370.

\title{
توزيع الأصفار لحلول معادلة تفاضلية محايدة من الدرجة الأولى
}

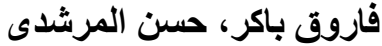 \\ قسم الرياضيات ـ كلية العلوم - جامعة دمياط - مصر
}

سوف نناقش مسألة تحديد المسافة بين الأصفار المتجاورة لجميع الحلول لمعادلة تفاضلية ذات معامل تأخير من الثكل

$$
[x(t)-p x(t-\tau)]^{\prime}+Q(t) x(t-\sigma)=0, \quad t \geq t_{0},
$$

حيث

$p>1, \tau, \sigma>0$

$Q \in C\left(\left[t_{0}, \infty\right),(0, \infty)\right.$

قدمنا الثروط الكافية للتنبذب لهذا النوع من المعادلات. وأعطينا بعض الأمثلة لتوضيح نتائجنا مع مقارنتها 